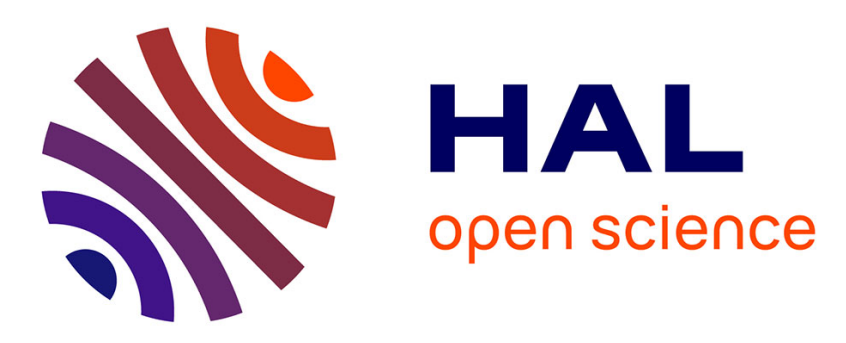

\title{
Characteristic times in transient Thermal Elasto-Hydrodynamic line contacts
}

Jonathan Raisin, Nicolas Fillot, David Dureisseix, Philippe Vergne, Vincent Lacour

\section{- To cite this version:}

Jonathan Raisin, Nicolas Fillot, David Dureisseix, Philippe Vergne, Vincent Lacour. Characteristic times in transient Thermal Elasto-Hydrodynamic line contacts. Tribology International, 2015, 82 (Part A), pp.472-483. 10.1016/j.triboint.2014.02.022 . hal-01225275

\section{HAL Id: hal-01225275 \\ https://hal.science/hal-01225275}

Submitted on 13 Mar 2018

HAL is a multi-disciplinary open access archive for the deposit and dissemination of scientific research documents, whether they are published or not. The documents may come from teaching and research institutions in France or abroad, or from public or private research centers.
L'archive ouverte pluridisciplinaire HAL, est destinée au dépôt et à la diffusion de documents scientifiques de niveau recherche, publiés ou non, émanant des établissements d'enseignement et de recherche français ou étrangers, des laboratoires publics ou privés. 


\title{
Characteristic times in transient Thermal Elasto-Hydrodynamic line contacts
}

\author{
J. Raisin ${ }^{\mathrm{a}, \mathrm{b}, *}$, N. Fillot ${ }^{\mathrm{a}}$, D. Dureisseix ${ }^{\mathrm{a}}$, P. Vergne ${ }^{\mathrm{a}}$, V. Lacour ${ }^{\mathrm{b}}$ \\ ${ }^{a}$ Université de Lyon, CNRS INSA-Lyon, LaMCoS UMR5259, F-69621 Villeurbanne, France \\ ${ }^{b}$ Total Supply Marketing - SDR, Centre de Recherche de Solaize, BP 22, Chemin du Canal, 69360 Solaize, France
}

This is a preprint of the article published in Tribology International 82:472-483, 2015. (C) 2015, Elsevier.

DOI: 10.1016/j.triboint.2014.02.022

\begin{abstract}
The paper presents a numerical and analytical analysis on transient effects in Thermal Elasto-Hydrodynamic (TEHD) contacts subjected to fluctuations of their operating conditions. Firstly, the system of equations, boundary conditions and numerical scheme used in the model are described. In addition, developments to solve transient TEHD problems are detailed, on the basis of a previous work [1] dedicated to the study of steady state cases. Then, the phenomena at the origin of transient effects in TEHD contacts are reviewed along with their characteristic time. In this context, a particular focus is placed on the thermal contribution. The complexity in finding a relevant thermal characteristic time is illustrated by the influence of the slide-to-roll ratio (SRR) on the contact performance (film thickness and friction). The dependency of the dominant heat transfer mode on the SRR is showed. A distinction between low sliding, pure sliding and high sliding conditions is made. Each configuration is thoroughly analyzed, leading to the formulation of new thermal characteristic times. Finally, time-dependent TEHD computations are performed in order to investigate the onset of transient effects. A direct relationship with the dominant thermal time is established and validated through a parametric study on the contact operating conditions and on the solid material properties.

Numerical simulation of transient Thermal Elasto-Hydrodynamic (TEHD) systems has several interests: understanding local behavior to prevent failure of a designated system, working conditions, fluid properties, overall design, etc. For practical applications, taking into account the thermal effects is mandatory, and since time-dependent operating conditions often apply, the decision to use a quasisteady or a transient model is also required. In this article, we derive, a priori, characteristic time scales for a generic line contact TEHD problem, and discuss the discrepancies between the two models depending on the dynamical character of the problem. A decision criteria is proposed and tested on various conditions to assess its validity.
\end{abstract}

Keywords: Thermal Elasto-Hydrodynamic Lubrication (TEHL), line contact, non-Newtonian fluids, transient effects, characteristic times, numerical simulation

\section{Introduction}

During their life cycle, complex tribological systems such as gears and cam-followers are subjected to extremely severe operating constraints. Those invariably involve substantial shear rates, pressures and temperatures within the lubricant, in addition to dynamical conditions of load, speeds and conjunction geometry [2]. Early performance assessment of these mechanical contacts consisted in applying semi-analytical formulas from the stationary Elasto-Hydrodynamic Lubrication (EHL) theory to determine critical film thicknesses [3]. Following the work of Vichard [4], several authors [5-8] then focused on transient effects and exemplified, e.g., the role of the squeeze-film term in preventing failure during reversal of entrainment. Although capable of precisely capturing the transient behavior, the aforementionned studies were based on two questionnable assumptions: i) a Newtonian rheology for the lubricant, which may produce rather satisfying solutions in terms of film thicknesses [9, 10], but was proved to greatly overestimate friction; ii) isothermal conditions, which are far from being valid in the

\footnotetext{
${ }^{*}$ Corresponding author

Email address: jonathan.raisin@insa-lyon.fr (J. Raisin)
}

presence of high sliding and/or entrainment velocities [11] typically found in these contacts.

Despite the emergence of shear-rate dependent viscosity $[12,13]$ and thermal models [14, 15] in the late 1970s and early 1980s, further progress remained severely hindered by computational power limitations for almost a decade. Since the 1990s, the development of more efficient methods, such as the multilevel multi-integration (MLMI) [16] and multigrid (MG) techniques [17], has allowed to significantly improve the relevance of the numerical models. In 1997, Larsson [18], e.g., presented a transient non-Newtonian EHL analysis of an involute spur gear. By comparing two lubricants with different rheological behaviors, the author highlited the importance of nonNewtonian effects in the prediction of friction. Later, Wang and Yang [19] included thermal effects in a study of an eccentrictapet pair subjected to a transient load. Their work confirmed the outstanding influence of temperature not only on friction, but also on the critical film thickness. Indeed, the latter (occuring at zero entrainment speed i.e. when both contacting surfaces move in opposite directions with the same magnitude) was found to originate from both the thermal viscosity wedge $[11,20]$ and the squeeze effects.

In recent years, the use of transient Thermal-Elasto- 
Hydrodynamic (TEHD) models is progressively becoming a standard for the simulation of gears and cam-follower systems. New advances focus on topics such as roughness, starvation, boundary lubrication, etc. Bobach et al. [21], e.g., incorporated mixed friction effects into a study on involute spur gears, revealing the importance of considering the topographies of the contacting surfaces. Surprisingly, a clear understanding of the onset and magnitude of the transient effects in TEHD configurations is still lacking. This paper aims at addressing this point by providing a comprehensive numerical and analytical analysis of the transient phenomena occuring within the conjunction, their respective influence (depending on the operating conditions and material parameters), and their associated characteristic times.

\section{Model description}

The numerical model described hereafter is based on the full system approach (FSA) used by Doki-Thonon [22] and originally developed by Habchi $[1,23]$. It is dedicated to the simulation of transient fully-flooded EHL problems, possibly involving thermal and non-Newtonian effects. Line contact geometries and smooth body surfaces are assumed (Figure 1).

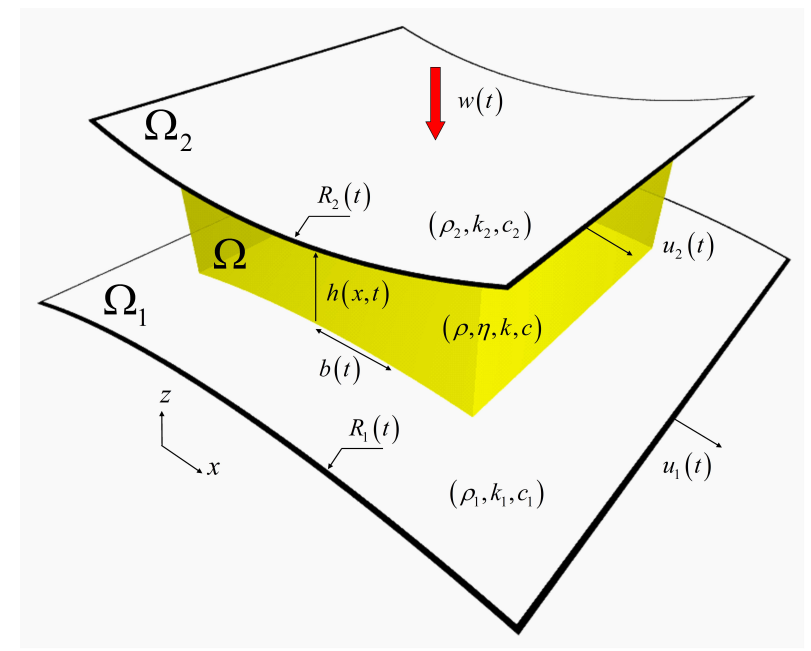

Figure 1: Schematic representation of a typical transient TEHL line contact between solids $\Omega_{1}$ and $\Omega_{2} . \Omega$ is the fluid domain.

\subsection{Governing equations}

\subsubsection{Fluid dynamics}

Transient generalized Reynolds equation. Fluid flow within the conjunction is governed by the transient generalized Reynolds equation introduced by Yang and $\mathrm{Wen}^{1}$ [24]:

$$
\frac{\partial}{\partial x}\left[\left(\frac{\rho}{\eta}\right)_{e} \frac{\partial p(x, t)}{\partial x}\right]-\frac{\partial}{\partial x}\left(\rho^{*}\right)-\frac{\partial}{\partial t}\left(\rho_{e}\right)=0
$$

where $p, \rho$ and $\eta$ stand for the pressure, density and viscosity of the lubricant, respectively. Other quantities in equation (1), read:

\footnotetext{
${ }^{1}$ Note that the retained form is actually that of Habchi et al. [1]
}

$$
\begin{aligned}
\left(\frac{\rho}{\eta}\right)_{e} & =\frac{\eta_{e}}{\eta_{e}^{\prime}} \rho_{e}^{\prime}-\rho_{e}^{\prime \prime} \\
\rho^{*} & =\rho_{e}^{\prime} \eta_{e}\left(u_{2}(t)-u_{1}(t)\right)+\rho_{e} u_{1}(t)
\end{aligned}
$$

where $u_{1}$ and $u_{2}$ are the velocities of the contacting bodies along the $x$ direction. Variables with the subscript ()$_{e}$ denote equivalent terms taking into account the variations of the physical lubricant properties across the film thickness, $h$. These terms, arising from the integration of the Navier-stokes equations, are expressed as:

$$
\begin{aligned}
\rho_{e} & =\int_{0}^{h} \rho d z \\
\rho_{e}^{\prime} & =\int_{0}^{h} \rho\left(\int_{0}^{z} \frac{1}{\eta} d z^{\prime}\right) d z \\
\rho_{e}^{\prime \prime} & =\int_{0}^{h} \rho\left(\int_{0}^{z} \frac{z^{\prime}}{\eta} d z^{\prime}\right) d z \\
\frac{1}{\eta_{e}} & =\int_{0}^{h} \frac{1}{\eta} d z \\
\frac{1}{\eta_{e}^{\prime}} & =\int_{0}^{h} \frac{z}{\eta} d z
\end{aligned}
$$

The film thickness depends on, $h_{0}$, the spacing of the rigid solids, $R$, the equivalent radius of curvature representing the original geometry and, $v$, the total elastic deflection [1], as:

$$
h(x, t)=h_{0}(t)+\frac{x^{2}}{2 R(t)}-v(x, t)
$$

As for boundary conditions, ambient pressure is prescribed at the inlet and outlet of the fluid domain. The corresponding abscissas, $x_{\text {in }}$ and $x_{\text {out }}$, are respectively located at $x=-5.4 b_{\text {ref }}$ and $x=3.6 b_{\text {ref }}, b_{\text {ref }}$ being the Hertzian contact half-width at the reference instant, $t_{\text {ref. }}$ Such values are similar to those classicaly found in the litterature for TEHD line contacts [1, 11, 19, 25]. Finally, asymmetry of the fluid domain is here justified by the fact that the entrainment was kept unidirectional in all the computed cases.

Density. The lubricant retained in this study is a fluid with properties typical of automotive engine oils (see Table 1). Its density is chosen to follow Dowson-Higginson's law [3]:

$$
\rho(p, T)=\rho_{r}\left(\frac{5.9 \times 10^{8}+1.34 p}{5.9 \times 10^{8}+p}-\beta_{D H}\left(T-T_{r}\right)\right)
$$

where $\beta_{D H}$ is the density-temperature coefficient and $\rho_{r}$ is the density measured at the reference temperature ${ }^{2}, T_{r}$.

\footnotetext{
${ }^{2}$ Not to be confused with, $T_{0}$, the initial temperature and corresponding properties used in the dimensionless model (see section 2.2).
} 
Rheology. In mechanical contacts operating under (T)EHD conditions, an accurate description of the viscosity variations is paramount. Among the numerous constitutive relations found in the litterature, those with higher physical relevance and a wider range of validity were favored in this study. The pressure and temperature dependence of the lubricant Newtonian viscosity $\mu$ were thus chosen to obey the modified WLF model [26]:

$$
\begin{aligned}
\mu \frac{-C_{1}\left(T-T_{g}(p)\right) F(p)}{C_{2}+\left(T-T_{g}(p)\right) F(p)} & \\
\text { with } T_{g}(p) & =T_{g}\left(p_{0}\right)+A_{1} \ln \left(1+A_{2} p\right) \\
F(p) & =1-B_{1} \ln \left(1+B_{2} p\right)
\end{aligned}
$$

where $\mu_{g}$ is the viscosity at the glass transition temperature $T_{g}, p_{0}$ is the ambient pressure and $A_{1}, A_{2}, B_{1}, B_{2}, C_{1}$ and $C_{2}$ are constants characterizing the fluid.

The generalized non-Newtonian viscosity is then obtained from Bair's modified version of the Carreau-Yasuda formulation [27]:

$$
\eta(p, T, \tau)=\frac{\mu(p, T)}{\left[1+\left(\frac{\tau}{G}\right)^{a}\right]^{\frac{1}{n}-1}}
$$

where $\tau=|\eta \partial u / \partial z|$ is the equivalent shear stress, $G$ is the shear modulus and $a$ and $n$ are additional constants characterizing the fluid.

\subsubsection{Solid mechanics}

A remarkable asset of Habchi's work [1] lies in the resolution of the structural mechanics problem. Firstly, by solving the classical linear elasticity equations with the finite element method rather than relying on the half-space assumption, he opened the way towards the modelling of more realistic contact configurations (as e.g. in complex kinematic EHD cases). Secondly, by applying the equivalent body theory, he managed to subsequently reduce the computational power requirements. The total elastic deflection $v$ (equation (2)) is deduced from the calculation of the displacement vector $\mathbf{U}$ on only one solid $\Omega_{1}$ (the other being considered rigid) of size $60 b_{\text {ref }} \times 60 b_{\text {ref }}$ [1], with equivalent properties (Young's modulus $E$ and Poisson's ratio $v$ ), such that:

$$
-\nabla \cdot \sigma=0
$$

with $\sigma=C \epsilon_{s}(\mathbf{U})$ where $C$ and $\epsilon_{s}$ are the stiffness and strain tensors, respectively. Regarding boundary conditions, null displacements are assumed on the bottom surface while side surfaces are left free. Finally, a downward vertical stress resulting from the lubricant pressure is prescribed at the fluid/solid interface.

\subsubsection{Load balance equation}

The last part of the EHD coupling consists in ensuring that the dynamical applied load, $w(t)$, is fully carried by the lubricant film. This is performed by solving the load balance equation on the fluid domain $\left(x \in\left[x_{\text {in }}, x_{\text {out }}\right]\right)$ :

$$
\int_{x_{\text {in }}}^{x_{\text {out }}} p(x, t) d x=w(t)
$$

\subsubsection{Energy conservation}

Neglecting body forces and heat radiation, the general form of the energy equation under transient regime writes [28]:

$$
\rho c\left(\frac{\partial T}{\partial t}+(\mathbf{u} \cdot \nabla) T\right)-\nabla \cdot(k \nabla T)=Q
$$

where $k, c$ and $T$ are the conductivity, specific heat capacity at constant pressure and temperature of the considered medium and $Q$ contains heat sources.

Within the fluid, heat advection in the $\mathrm{z}$-direction is neglected following the thin-film assumption used to model the lubricant flow. Futhermore, in view of the typical Peclet numbers $P e=\rho u_{m} b c / k\left(u_{m}=\left(u_{1}+u_{2}\right) / 2\right.$ and $b$ respectively being the entrainment velocity and characteristic length scale), advection clearly dominates over diffusion in the x-direction. The fluid heat equation thus reduces to:

$$
\begin{aligned}
& \rho c\left(u(x, z, t) \frac{\partial T}{\partial x}+\frac{\partial T}{\partial t}\right)-\frac{\partial}{\partial z}\left(k \frac{\partial T}{\partial z}\right)= \\
& -\frac{T}{\rho} \frac{\partial \rho}{\partial T}\left(u(x, z, t) \frac{\partial p}{\partial x}+\frac{\partial p}{\partial t}\right)+\eta\left(\frac{\partial u(x, z, t)}{\partial z}\right)^{2}
\end{aligned}
$$

with

$$
\begin{aligned}
u(x, z, t) & =\frac{\partial p}{\partial x}\left(\int_{0}^{z} \frac{z}{\eta} d z-\frac{\eta_{e}}{\eta_{e}^{\prime}} \int_{0}^{z} \frac{1}{\eta} d z\right) \\
& +\eta_{e}\left(u_{2}(t)-u_{1}(t)\right) \int_{0}^{z} \frac{1}{\eta} d z+u_{1}(t)
\end{aligned}
$$

The first and second right-hand side terms in equation (9) account for the production of heat by compression and shearing, respectively.

Within the solid bodies, previous considerations regarding dominant heat transfer mode globally apply. Still, diffusion along the $\mathrm{x}$-direction should not be neglected here as it is likely to play a role in configurations close to pure sliding (see section 3.5 and [11]). Finally, since no heat sources is present, the solid heat equations are expressed as:

$$
\begin{gathered}
\rho_{i} c_{i}\left(u_{i}(t) \frac{\partial T}{\partial x}+\frac{\partial T}{\partial t}\right)-\frac{\partial}{\partial x}\left(k_{i} \frac{\partial T}{\partial x}\right)-\frac{\partial}{\partial z}\left(k_{i} \frac{\partial T}{\partial z}\right)=0 \\
i=1,2
\end{gathered}
$$

Consistency of the full thermal problem is ensured by imposing the continuity of the heat flux and temperature accross 
the fluid/solid interfaces $\delta_{\Omega_{i}}$ along with proper boundary conditions. At the inlet and outlet of the fluid domain, conditional relations are prescribed such that lubricant entering the contact does so at the ambient temperature $T_{0}$ :

$$
\left.T\right|_{x_{i n}, z, t}=T_{0} \text { if } u(z, t)>0,\left.T\right|_{x_{\text {out }}, z, t}=T_{0} \text { if } u(z, t)<0
$$

Similarly, equations (11) require conditions able to accomodate possible changes in the direction of movement of the solids:

$$
\left.T\right|_{x_{i n}, z, t}=T_{0} \text { if } u_{i}(t)>0,\left.T\right|_{x_{\text {out }}, z, t}=T_{0} \text { if } u_{i}(t)<0
$$

Far from the contact area, i.e. at a depth of about ${ }^{3} 3 b_{\text {ref }}$ inside the solids $[1,11,19,22]$ a zero conductive heat flux is applied.

\subsection{Numerical procedure}

For the sake of computational efficiency and versatility, all the above equations and their respective boundary conditions were first transformed in their dimensionless form using the following quantities:

$$
\begin{gathered}
\bar{x}=\frac{x}{b_{\mathrm{ref}}}, \bar{z}= \begin{cases}z / h, & \text { in the lubricant } \\
z / b_{\mathrm{ref}}, & \text { in the solids }\end{cases} \\
R_{t}=\frac{R(t)}{R_{\mathrm{ref}}}, W_{t}=\frac{w(t)}{w_{\mathrm{ref}}}, U_{m t}=\frac{u_{2}(t)+u_{1}(t)}{2 u_{m_{\mathrm{ref}}}} \\
\bar{p}=\frac{p}{p_{h_{\mathrm{ref}}}}, H=\frac{h R_{\mathrm{ref}}}{b_{\mathrm{ref}}^{2}}, \bar{\rho}=\frac{\rho}{\rho_{0}}, \bar{\mu}=\frac{\mu}{\mu_{0}}, \bar{t}=\frac{t u_{m_{\mathrm{ref}}}}{b_{\mathrm{ref}}}
\end{gathered}
$$

where the superscript () ref denotes the instant chosen as reference, $t_{\text {ref }}$. In practice, a special attention is paid on the choice of $t_{\text {ref }}$ to ensure a proper scaling of the model whatever the evolutions of the operating conditions. The equations were then implemented in a commercial finite element code [29]. The basic features of the present model were directly derived from Habchi's. Therefore, readers looking for additional details on, e.g., the mesh structuration, the type and order of shape functions and the handling of the cavitation region should refer to $[1,23]$. Emphasis is here placed on the main modifications of the architecture introduced to solve transient TEHD problems. A significant change concerns the computation of the integral terms in equation (1) and the generalized viscosity $\eta$ within the fluid film. Those are no longer obtained through external algorithms, but rather as solution of three additional partial differential equations (PDEs). For the integral terms, the PDEs write:

$$
\begin{aligned}
& \frac{\partial}{\partial z}(f)-\frac{1}{\eta}=0 \\
& \frac{\partial}{\partial z}(g)-\frac{z}{\eta}=0
\end{aligned}
$$

\footnotetext{
${ }^{3}$ In pure sliding conditions, this distance is doubled for the stationnary solid.
}

where $f$ and $g$ respectively give the value of $\int_{0}^{z}(1 / \eta) d z$ and $\int_{0}^{z}(z / \eta) d z$ at any point $M(x, z)$ of the fluid domain. As for the generalized viscosity, $\eta(p, T, \tau) 5$, the circular dependency with the equivalent shear stress, $\tau=|\eta \partial u / \partial z|$, is simply avoided through the resolution of:

$$
\eta-j=0
$$

where $j$ stands for any formulation retained to model the rheological behavior of the lubricant.

An advantage of this method is that all the TEHD equations are now combined into a single non-linear system ensuring the full coupling [11]. The system is then solved using a NewtonRaphson procedure.

The numerical scheme for the simulation of transient TEHD problems consists of two phases: the initialization and the resolution itself (Figure 2). A distinction is made here since common analytical solutions fail at providing a consistent initial guess for all the variables. Indeed, a very accurate prediction of the complex outcome of the competing physical phenomena acting at the initial state is required. Initialization is thus achieved through a stationary TEHD computation, under the following steps: at first, solid deformations corresponding to a Hertzian pressure profile are pre-calculated. Then, the cor-

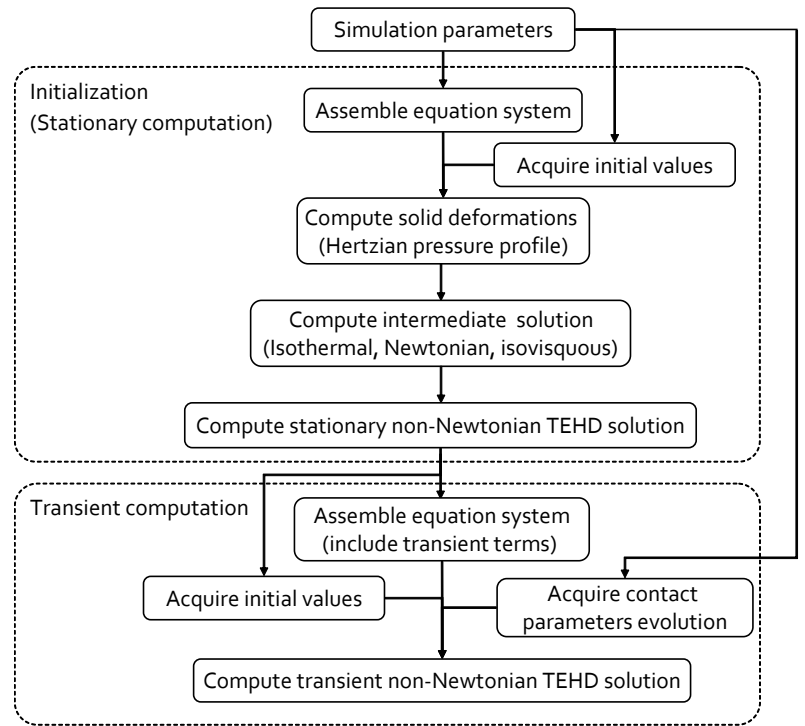

Figure 2: Schematic flowchart for the transient TEHD computations.

responding isothermal Newtonian isoviscous elastic problem is solved. This intermediate step has the advantage of eliminating infinite values of the longitudinal pressure gradient causing issues in the shear rate calculation. It also allows to avoid the consideration of piezoviscous effects, costly and unnecessary at this point. Finally, the fully coupled stationary TEHD problem is computed. From there, obtaining the transient evolution is pretty much straightforward (see Figure 2). After compilation of the set of equations, the resulting differential-algebraic system is discretized through the method of lines and solved using 
a variable step size variable order ${ }^{4}$ backward differentiation formula (BDF) scheme. Time steps are automatically adjusted according to the relative and absolute tolerances specified $(0.001$ and 0.0001 , respectively). Typical transient TEHD computations (consisting of 1366, 13632, 75602 and 25211 degrees of freedom for the fluid, elastic, non-Newtonian and thermal problems, respectively, and of 2000 saved time steps) performed in this study last about 5 hours and 30 minutes (10 minutes for the initialization) on a computer equipped with an Intel Core i7-2620M processor.

\section{Origin of transient effects and associated characteristic times}

A wide variety of tribological systems are commonly subjected to fluctuations of the operating conditions. Such fluctuations can be the result of vibrations or simply due to the nature of the application, as in gears and cam-followers. In any case, those can dramatically affect the tribological performances (film thickness and friction) if their rate of occurence exceeds the rate at which the physical phenomenon activated in the conjunction can accomodate. In this section, the involved characteristic times are reviewed and discussed with respect to a reference configuration corresponding to the initial/steady state $t_{0}$ of the transient simulations of section 4 . Related operating conditions, lubricant and solid properties are detailed in Table $1)$.

\subsection{Operating fluctuation time}

Fluctuations can take the form of changes in the applied load, $w$, velocities, $u_{i}$, as well as in the contacting geometries, $R$. For the sake of clarity, only a sinusodal load variation superimposed to an initial/steady state value, i.e. $w=w_{0}\left[1+A_{w} \sin (\omega t)\right]$, is considered in the present study. The operating fluctuation time $t_{w}$ thus writes:

$$
t_{w}=\frac{2 \pi}{\omega}
$$

where $\omega$ is the load pulsation. Additionaly, note that the load amplitude $A_{w}$ was kept small enough to neglect the timedependency of the contact half-width, $b$, in the estimation of the other characteristic times. Although general formulations were retained in the following definitions, all numerical values (as e.g. those listed in Table 2) were computed using $b_{0}$ (corresponding to the initial load, $w_{0}$ ).

\subsection{Hydrodynamic time}

The hydrodynamic time, $t_{h}$, is a measure of how fast the contact will react in terms of film thickness. Indeed, perturbations generated at the inlet are transported by the lubricant flow (at the entrainement velocity, $u_{m}$ ) and arrive at the contact center after a delay:

$$
t_{h}=\frac{m}{u_{m}}
$$

\footnotetext{
${ }^{4}$ The time-stepping order is here resticted to 2 to prevent stability issues.
}

\begin{tabular}{|c|c|c|}
\hline \multicolumn{3}{|c|}{ PHYSICAL PROPERTIES OF THE LUBRICANT } \\
\hline$\rho_{r}$ & $\left(k g \cdot m^{-3}\right)$ & 837.2 \\
\hline$\beta_{D H}$ & $\left(K^{-1}\right)$ & $7 e-4$ \\
\hline$\mu_{g}$ & $\left(\right.$ Pa.s $\left.^{-1}\right)$ & $1 \mathrm{e} 12$ \\
\hline$A_{1}$ & $(K)$ & 19.648 \\
\hline$A_{2}$ & $\left(P a^{-1}\right)$ & $4.758 \mathrm{e}-9$ \\
\hline$B_{1}$ & $(-)$ & 0.213 \\
\hline$B_{2}$ & $\left(P a^{-1}\right)$ & $2.521 \mathrm{e}-8$ \\
\hline$C_{1}$ & $(-)$ & 15.798 \\
\hline$C_{2}$ & $(K)$ & 21.557 \\
\hline$T_{g}\left(p_{0}\right)$ & $(K)$ & 189 \\
\hline$T_{r}, T_{0}$ & $(K)$ & 298 \\
\hline$p_{0}$ & $(P a)$ & $1 \mathrm{e} 5$ \\
\hline$G$ & $(P a)$ & $7 \mathrm{e} 6$ \\
\hline$n$ & $(-)$ & 0.35 \\
\hline$a$ & $(-)$ & 5 \\
\hline$k$ & $\left(W \cdot m^{-1} \cdot K^{-1}\right)$ & 0.15 \\
\hline$c$ & $\left(J \cdot k^{-1} \cdot K^{-1}\right)$ & 2300 \\
\hline
\end{tabular}

Table 1: Reference configuration.

\begin{tabular}{llr}
\multicolumn{2}{l}{ PHYSICAL PROPERTIES OF THE SOLIDS } \\
\hline$E_{i}$ & $(\mathrm{~Pa})$ & $2 \mathrm{e} 11$ \\
$v_{i}$ & $(-)$ & 0.3 \\
$\rho_{i}$ & $\left(\mathrm{~kg} \cdot \mathrm{m}^{-3}\right)$ & 7850 \\
$k_{i}$ & $\left(\mathrm{~W} \cdot \mathrm{m}^{-1} \cdot \mathrm{K}^{-1}\right)$ & 47 \\
$c_{i}$ & $\left(\mathrm{~J} . \mathrm{kg}^{-1} \cdot \mathrm{K}^{-1}\right)$ & 450 \\
\hline
\end{tabular}

\begin{tabular}{llr}
\multicolumn{2}{l}{ CONTACT PARAMETERS } \\
\hline$R_{0}$ & $(\mathrm{~m})$ & 0.01 \\
$w_{0}$ & $\left(N . \mathrm{m}^{-1}\right)$ & $2 \mathrm{e} 5$ \\
$u_{m_{0}}$ & $\left(m . \mathrm{s}^{-1}\right)$ & 2.5 \\
$E_{r}$ & $(\mathrm{~Pa})$ & $2.198 \mathrm{e} 11$ \\
$v_{r}$ & $(-)$ & 0.3 \\
$b_{0}$ & $(\mathrm{~m})$ & $1.522 \mathrm{e}-4$ \\
$p_{h_{0}}$ & $(\mathrm{~Pa})$ & $8.364 \mathrm{e} 8$ \\
\hline
\end{tabular}

where $m$ is the distance traveled. Expressing the latter is challenging as it requires an accurate knowledge of the inlet location, which varies with the operating conditions [30]. In a series of paper on the amplitude reduction of surface waviness in contacts operating in the piezoelastic regime, Hooke and Venner $[31,32]$ extrapoled the following relationship:

$$
m=b M_{\mathrm{MV}}^{-1 / 2} L_{\mathrm{MV}}^{1 / 2}
$$

Providing the validity condition is satisfied, equation (17) gives values around unity ( $m=1.35 b_{0}$ in the reference configuration). In this regard, a distance of one contact half-width, $b$, was retained to approximate the distance traveled by the film thickness perturbations leading to $t_{h}=b / u_{m}$.

\subsection{Elastic time}

The elastic time, $t_{e}$, represents the rate at which the deformation of the solids adapts to a change in the lubricant pressure. A classical formulation for $t_{e}$ is given by:

$$
t_{e}=\frac{L}{\lambda \sqrt{\frac{E_{i}}{\rho}}} \text { with } \lambda \sim 1
$$

where $L$ is a characteric length (typically the contact halfwidth, $b$ ) and $\lambda \sqrt{E_{i} / \rho}$ is a fair approximation of the propagation 
velocity of waves in an elastic material $[33,34]$. In practice, $t_{e}$ is small enough compared to the other characteristic times so that the transient solid mechanics problem can be considered as a succession of stationary states.

\subsection{Rheological time}

The rheological time, $t_{\dot{\gamma}}$, describes the transient response of non-Newtonian lubricants subsequently to shear rate variations within the fluid flow. A parallel can be made with the phenomenon of shear relaxation of polymers suspended in solvants. According to the kinetic theory of polymeric liquids [35], the corresponding characteristic time, $t_{\dot{\gamma}}$, is expressed as:

$$
t_{\dot{\gamma}}=\frac{\mu(p, T)}{G}
$$

with the fluid low-shear viscosity, $\mu(p, T)$, being evaluated within the pressurized region (here as an average value over the film thickness at the contact center). Because lubricants exhibit a wide range of non-Newtonian behavior, $t_{\dot{\gamma}}$ found in TEHD contacts span over several order of magnitude. A weakly non-Newtonian fluid, i.e. with a negligible $t_{\dot{\gamma}}$, was deliberately picked in this study in accordance with the use of a stationnary formulation to model the shear-viscosity dependency (see equation (5)).

\subsection{Thermal time}

The choice of a relevant thermal characteristic time in TEHD contacts is a less documented and much more complex problem. Indeed, identifying the dominant heat transfer modes with regard to the operating conditions requires a deep understanding of the energy balance within the conjunction (see Figure 3). In this respect, Bruyere et al. [11] exemplified that the

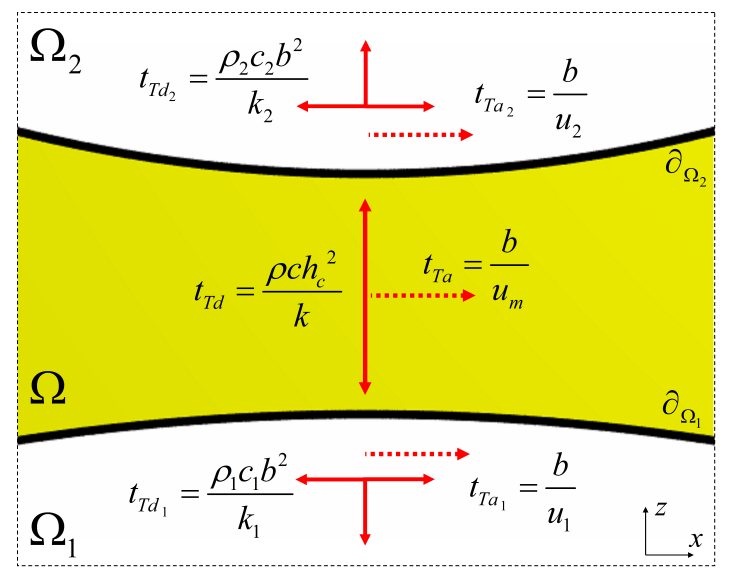

Figure 3: Schematic representation of the energy balance in a typical TEHD line contact (here operating under low sliding). Heat transfers by diffusion and advection (respectively depicted by plain and dotted red arrows) are reported with their associated characteristic times.

slide-to-roll ratio (SRR) has a crucial and highly non-trivial influence. A series of stationary TEHD computations were thus performed using the reference configuration (see Table 1) and different SRRs to illustrate the point. Deduced values for the central $\left(h_{c}\right)$ and minimum $\left(h_{m}\right)$ film thicknesses and for the friction coefficient $\left(C_{f}\right)$ are plotted on Figures 4 and 5, respectively, and compared to isothermal solutions. $C_{f}$ is here defined as the average of the friction forces (per unit length) calculated at both fluid/solid interfaces divided by the applied load:

$$
C_{f}=\frac{1}{2 w} \sum_{i=1}^{2}\left|\int_{\delta_{\Omega_{i}}} \tau_{z x} d x\right|
$$

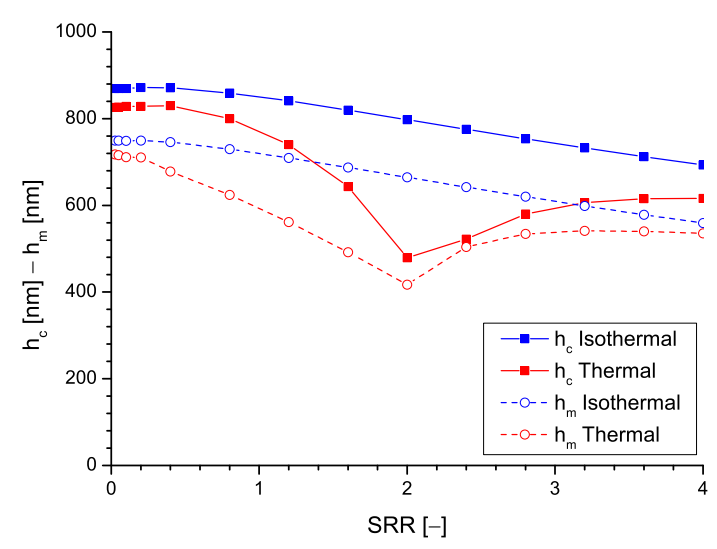

Figure 4: Variation of the central and minimum film thicknesses $\left(h_{c}\right.$ and $h_{m}$, respectively) as a function of the slide-to-roll ratio (SRR) for the reference configuration (see Table 1).

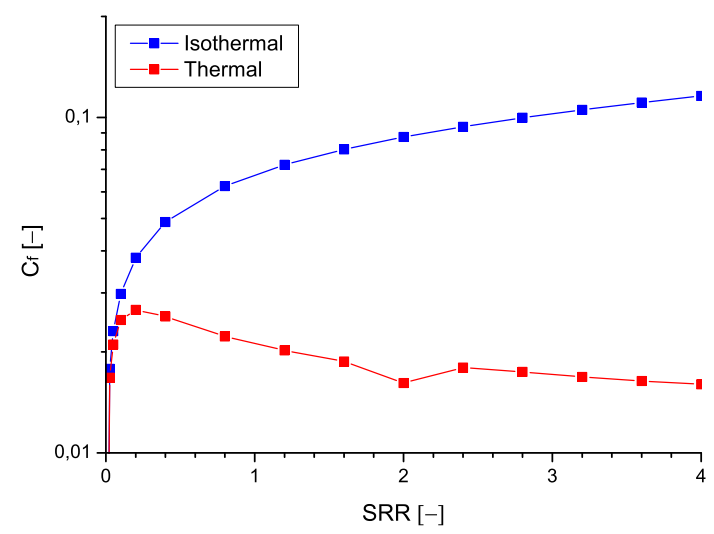

Figure 5: Variation of the friction coefficient as a function of the slide-to-roll ratio (SRR) for the reference configuration (see Table 1).

In agreement with previous results [11], present ones show that thermal effects arising with the increase in the slide-toroll ratio have large consequences on both the film thickness and friction coefficient. Above all, those no longer follow the monotonous variations predicted with the isothermal assumption but rather experience a local minimum in pure sliding conditions $(S R R=2)$. Three different regimes, namely low, pure and high sliding must therefore be distinguished. Note that a 


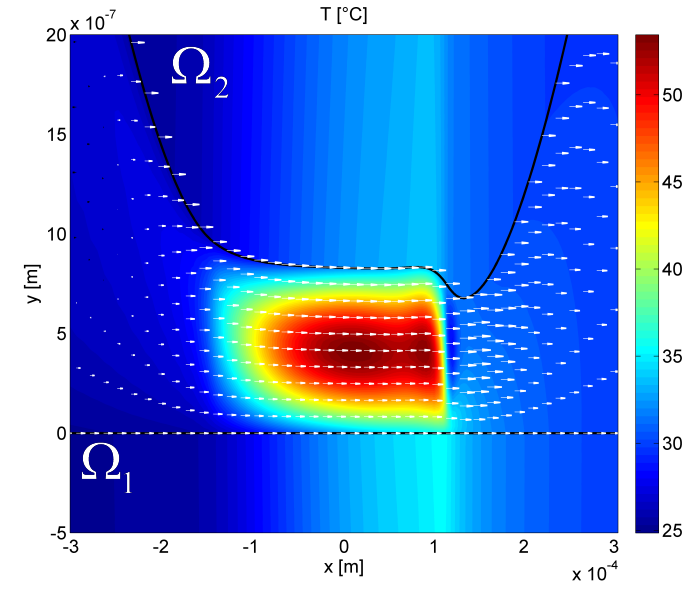

Figure 6: Temperature (color) and velocity (arrows) fields in the lubricant at low sliding case $(S R R=0.4)$.

comprehensive study on friction in TEHD (point) contacts at very low SRRs is available in [36].

Low sliding $(S R R<2)$. At low sliding, thermal effects are moderate but still significant. As observed on Figure 6, lubricant temperature rises from $25^{\circ} \mathrm{C}$ up to about $53^{\circ} \mathrm{C}$. At the contact inlet (around $x=-b_{0}=-152.2 \mu \mathrm{m}$ ), shear and compression heating slightly lower the lubricant viscosity and consequently affect the central and minimum film thicknesses. Evidence of this mechanism is, for instance, illustrated by the discrepancies between the thermal and isothermal solutions close to pure rolling conditions on Figure 4. A first characteristic time is thus associated with the advection of thermally induced film thickness perturbations, $t_{T a}=t_{h}=b / u_{m}$. Yet, another heat transfer mechanism is believed to play a more crucial role. From both the temperature distributions (Figure 6) and friction results (Figure 5), it is clear that the largest amount of heat is generated within the pressurized region. Most of that heat diffuses along the film thickness (the Graetz number [37], $G z=\rho c u_{m} h_{c}^{2} /(k b)=t_{T d} / t_{T a}=0.021$, being smaller than unity). It then diffuses accross the fluid/solid interfaces and into the depth of the solids, before being finally transported outside of the contact (the Peclet number, $P e_{i}=\rho_{i} c_{i} u_{i} b / k_{i}=t_{T d_{i}} / t_{T a_{i}}$, for solids $\Omega_{1}$ and $\Omega_{2}$, respectively equal to $P e_{1}=22.89$ and $P e_{2}=34.33$, being larger than unity). Because different modes and directions of heat transfer are involved, finding a relevant characteristic time is far from straightforward. An obvious approach to the problem consists in comparing each one of them and identifying the limiting one. However, whereas $t_{T_{d}}=\rho c h_{c}^{2} / k$ and $t_{T a_{i}}=b / u_{i}$ seem to adequately represent the diffusion of heat within the lubricant and its advection by the solids, very little is known on the intermediate stage and its characteristic time. By looking at Figure 7, it is obvious that the contact half-width, $b$, does not accurately represent the depth over which the solid temperature is modified. Furthermore, it does not take into account the influence of the thermal properties of the contacting bodies (changed from steel to glass between Figures $7 \mathrm{a}$ and $7 \mathrm{~b}$ ). From both Figures, it appears

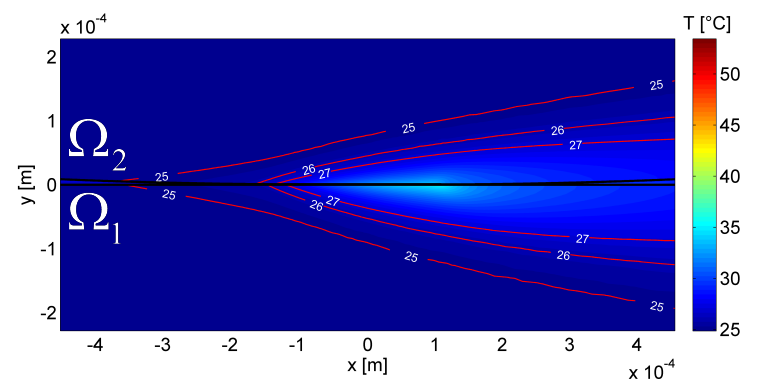

(a)

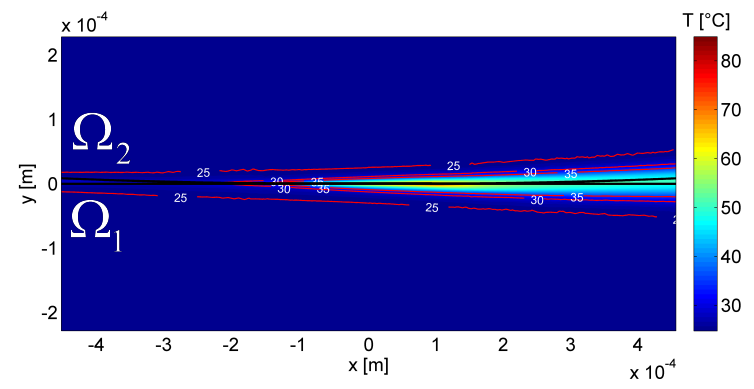

(b)

Figure 7: Temperature distribution (surface color and isolines) in the contacting bodies at low sliding $(S R R=0.4)$. Results from (a) the reference configuration (Table 1) and (b) obtained using the thermal properties of glass for the solids $\left(\rho_{i}=2530 \mathrm{~kg} \cdot \mathrm{m}^{-3}, c_{i}=720 \mathrm{~J} \cdot \mathrm{kg}^{-1} \cdot \mathrm{K}^{-1}, k_{i}=1 \mathrm{~W} \cdot \mathrm{m}^{-1} \cdot \mathrm{K}^{-1}\right)$.

that the characteristic length for the diffusion of heat accross the fluid/solid interfaces is the depth of the thermal boundary layer, $\delta_{T}$. In order to propose an expression for the latter, an analogy can be made with problems of forced convection in liquid metals [38]. In such problems, the depth of the hydrodynamic boundary layer, $\delta_{H}$, is negligible compared to that of the thermal boundary layer $\delta_{T}$. If, in addition, the velocity profile of the liquid metal flow is uniform, the energy equation used to model the heat transfer reduces to equation (11). By further exploiting the order of magnitude difference between the longitudinal and transverse temperature gradient (respectively $\partial T / \partial x$ and $\partial T / \partial z$ ) throughout the pressurized region (Figures $8 \mathrm{a}$ and $8 \mathrm{~b}$ ), it becomes possible to relate $\delta_{T}$ to the solids diffusivity, $k_{i} /\left(\rho_{i} c_{i}\right)$, velocity, $u_{i}$, and the characteristic length, $b$, over which heat is transferred from the lubricant [39]:

$$
\delta_{T}=\sqrt{\frac{k_{i} b}{\rho_{i} c_{i} u_{i}}}
$$

Using equation (21), the characteristic time for the diffusion of heat accross the fluid/solid interfaces and into the thermal boundary layer of the solids simplifies to:

$$
t_{T d_{e q_{i}}}=\frac{\rho_{i} c_{i} \delta_{T}^{2}}{k_{i}}=\frac{b}{u_{i}}
$$

which, as stated earlier, also represents the time scale for the advection of heat by the solids, $t_{T a_{i}}$. While unexpected, this result is of primary importance. Indeed, since at low sliding, one of the contacting bodies (here $\Omega_{1}$ ) moves slower than the lu- 


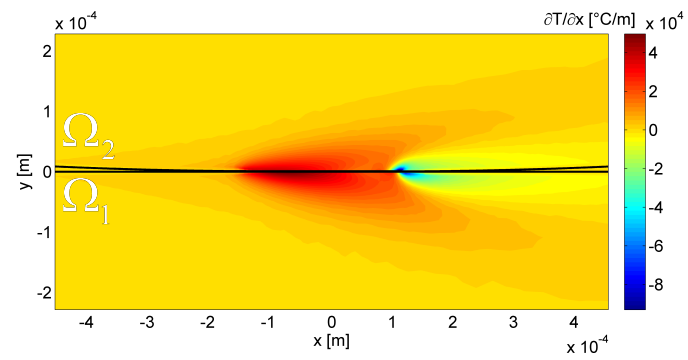

(a)

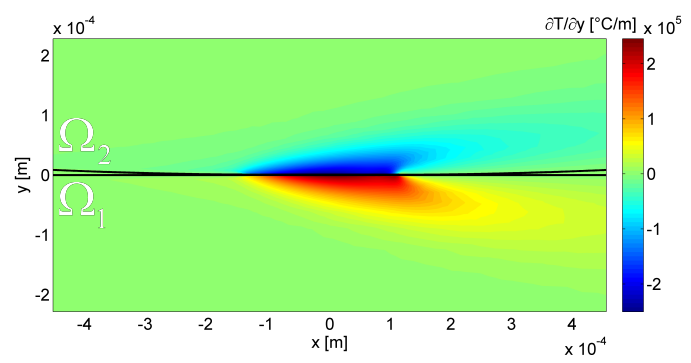

(b)

Figure 8: Longitudinal (a) and transverse (b) temperature gradient in the contacting bodies at low sliding $(S R R=0.4)$.

bricant entrainment velocity (here $\Omega_{1}$ ), a comparison between the different thermal characteristic times will always yield (provided $P e_{i} \gg 1$ ): $t_{T d_{e q_{i}}}=t_{T a_{i}}>t_{T a}>t_{T d}$. In other words, it implies that the onset of transient thermal effects is likely to be independent of the solids (thermal) properties.

Pure sliding $(S R R=2)$. In and close to pure sliding conditions, the energy balance is completely altered (Figure 9 to compare to Figures 6 and 7a). The largest amount of the produced heat now exits the conjunction through the upper solid moving at twice the lubricant speed. The remaining heat gets trapped by the lower (stationnary) solid, significantly raising its temperature (up to almost $110^{\circ} \mathrm{C}$ ) on a large area, by diffusion (Figure 9b). As a consequence, lubricant flowing in the lower solid vicinity is heated before reaching the contact inlet, reducing its viscosity and therefore the central and minimum film thicknesses (Figure 4). Friction is further decreased as the average lubricant temperature is very high in the whole pressurized region (Figure 9a). In this configuration, the dominant thermal characteristic time is associated with the diffusion of heat through the stationnary solid (here $\Omega_{1}$ ), from the contact centre to the inlet area: $t_{T d_{1}}=1742 \mu \mathrm{s} \gg t_{T a}=60.89 \mu \mathrm{s}$.

High sliding $(S R R>2)$. At high sliding, the heat flux distribution ressembles that observed at low sliding. However, because the solids move in opposite directions, strong differences in tribological performances exist (Figures 4 and 5). Indeed, at both the 'inlet' and 'outlet', lubricant at ambient temperature is dragged into the contact by one solid and is also ejected out after being heated by the other one [11]. Within the pressurized region, a stationary recirculation area where very high temperatures are reached (up to about $145^{\circ} \mathrm{C}$ on Figure 10) forms between the two 'colder' fluid layers. As a result

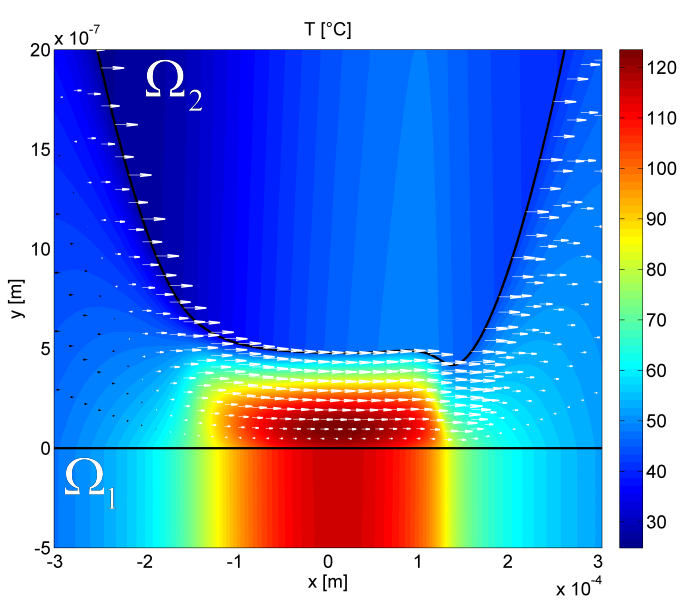

(a)

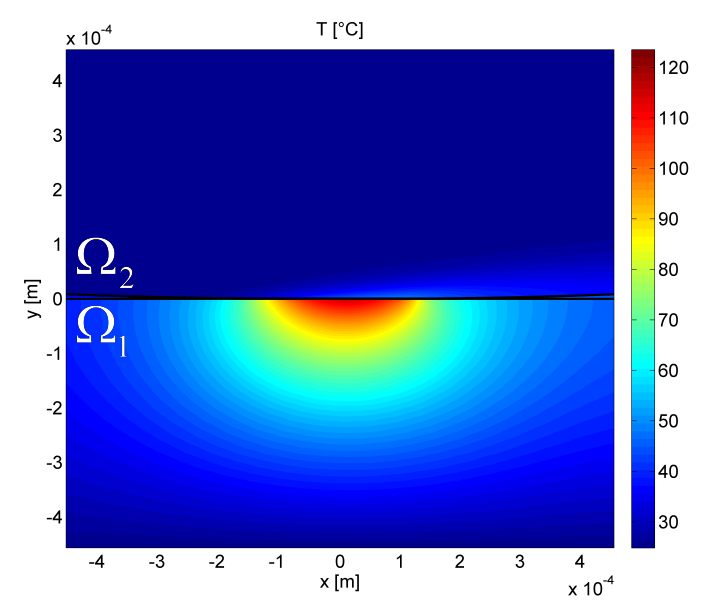

(b)

Figure 9: Temperature (color) and velocity (arrows) fields corresponding to a pure sliding case $(S R R=2)$.

from this differential heating, a viscosity gradient is created accross the film thickness which, in turn, modifies the pressure profile. This phenomenon, known as 'the viscosity wedge effect' [11], is responsible for the local film thickness increase in the central area of the contact (Figure 10). At high sliding, the dominant thermal characteristic time is associated with the advection of heat from the center of the contact back to the inlet: $t_{T a_{1}}=76.11 \mu \mathrm{s}>t_{T a}=60.89 \mu \mathrm{s}$.

\section{Onset of transient effects}

\subsection{Configuration}

In order to determine the onset of transient effects, timedependent TEHD computations were performed, in the reference configuration, for different values of the slide-to-roll ratio ([0.4, 1.7, 2, 2.4 3.6]) and varying load fluctuation frequencies, $t_{w}^{-1}$, ranging from $1 \mathrm{~Hz}$ to $30000 \mathrm{~Hz}$. For each combination of parameters, the transient response of the contact was monitored until reaching a periodic behavior (Figure 11). Usually, this requirement was fulfilled after at least 10 periods of load 


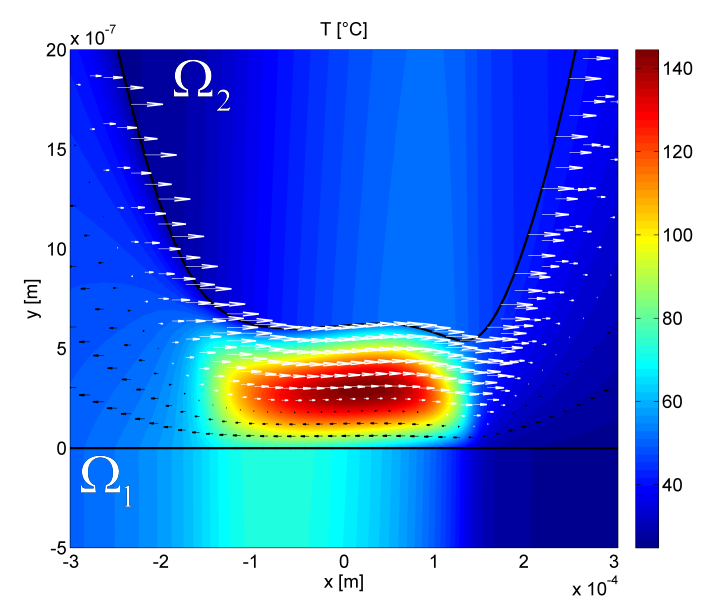

Figure 10: Temperature (color) and velocity (arrows) fields corresponding to a high sliding case $(S R R=3.6)$.

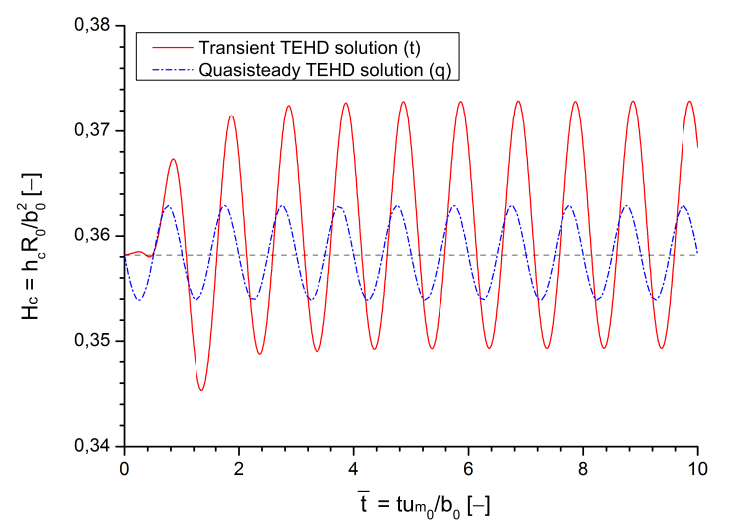

Figure 11: Transient and quasi-steady film thickness evolutions of a TEHD line contact subjected to a sinusoidal load variation. Reference configuration with $S R R=0.4$ and $\Omega_{h / w}=t_{h} / t_{w}=1$.

oscillation (more at high excitation frequencies, i.e. $t_{w} \ll t_{h}$ ). The transient evolution of the tribological performances, i.e. the central film thickness $\left(h_{c}\right)$ and friction coefficient $\left(C_{f}\right)$, were then extracted and compared to quasisteady results obtained by cancelling the time derivatives in equations (1), (9) and (11). For this purpose, characteristic variables, consisting of two mean values $\left(h_{c_{m}}, C_{f_{m}}\right)$ and one amplitude $\left(A_{h_{c}}\right)$ were built from each simulation. Those were, in turn, used to create a new set of normalized variables, allowing to compute the deviations induced by the transient effects:

$$
\begin{aligned}
\Delta h_{c_{m}} & =\frac{h_{c_{m_{t}}}-h_{c_{m_{q}}}}{h_{c_{m_{q}}}}, \\
\Delta A_{h_{c}} & =\frac{A_{h_{c_{t}}}-A_{h_{c_{q}}}}{A_{h_{c_{q}}}}, \\
\Delta C_{f_{m}} & =\frac{C_{f_{m_{t}}}-C_{f_{m_{q}}}}{C_{f_{m_{q}}}},
\end{aligned}
$$

where the subscript ()$_{t}$ and ()$_{q}$ stand for transient and quasisteady results, respectively.

As a preliminary step, only the variation of the normalized mean value of the central film thickness $\Delta h_{c_{m}}$ as a function of the load fluctuation frequency $t_{w}^{-1}$ is considered. Figure 12

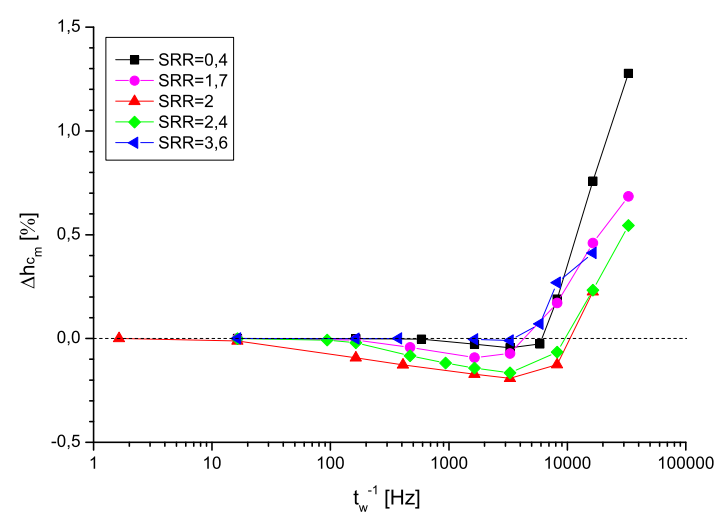

Figure 12: Influence of the transient effects on the variation of the normalized average central film thickness $\Delta h_{c_{m}}$ as a function of the load fluctuation frequency $t_{w}^{-1}$. Reference configuration with different SRRs.

shows that curves, representing different slide-to-roll ratios, follow similar trends. However, the onset above which the influence of transient effects is observed strongly differs from one $S R R$ to another (from $t_{w}^{-1} \sim 16 \mathrm{~Hz}$ for $S R R=2$ up to $t_{w}^{-1} \sim 590 \mathrm{~Hz}$ for $\left.S R R=0.4\right)$. Since only the dominant thermal characteristic time was varied in the simulations (the others being either constant $\left(t_{h}, t_{e}\right)$ or negligibly small $\left(t_{\dot{\gamma}}\right)$, it appears that the onset of transient effects is someway linked to the heat transfers within the conjunction.

\subsection{Results and discussion}

Based on the former observation, the variation of the normalized variables, $\Delta h_{c_{m}}, \Delta A_{h_{c}}$ and $\Delta C_{f_{m}}$, are plotted, on Figures 13, 14 and 15 respectively, as a function of, $\Omega_{T / w}$, the ratio of the dominant thermal time, $t_{T}$, and of the load fluctuation time, $t_{w}$. The former, specific to the different regimes of sliding identified in section 3.5, is recalled as $t_{T d_{e q_{1}}}$ for $S R R<2, t_{T d_{1}}$ for $S R R=2$ and $t_{T a_{1}}$ for $S R R>2$.

As expected, deviations induced by transient effects now all share the same appearance threshold $\left(\Omega_{T / w} \sim 0.029\right)$. In order to understand the underlying mechanism, one has to look closely at the characteristic times involved in the different simulations (Table 2). Indeed, independently of the $S R R$ considered, the dominant thermal characteristic time, $t_{T}$ is larger than the others. Therefore, as the frequency of the load fluctuation increases, thermal transfers are the first not able to accomodate. Then, as the threshold for the other phenomena (e.g. $\Omega_{h / w}=t_{h} / t_{w} \sim 0.029$ for hydrodynamics) are reached, they also start contributing significantly to the transient effects. Such a result is particularly interesting. Not only it validates the thermal analysis developed in the paper, but it also identifies thermal transfers as the most probable (i.e. most frequently encountered) source of transient effects in TEHD contacts. 


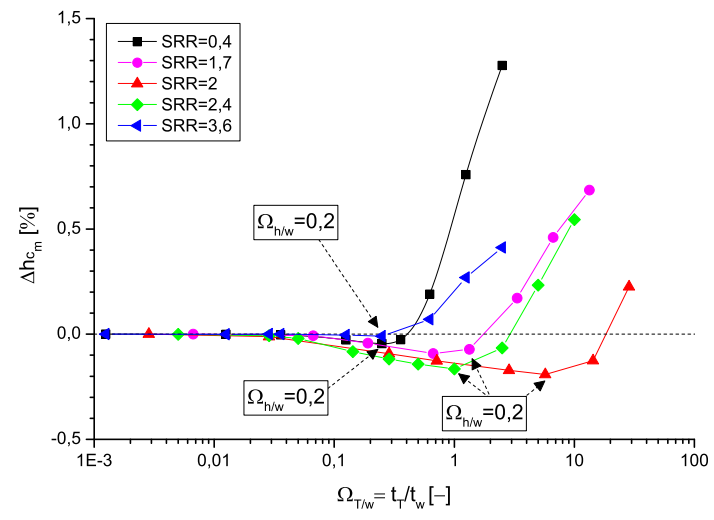

Figure 13: Influence of the transient effects on the variation of the normalized average central film thickness $\Delta h c_{m}$ as a function of the load fluctuation frequency $t_{w}^{-1}$. In abscissa, the latter is normalized by the dominant thermal time $t_{T}$ identified for each sliding regime $\left(t_{T d_{e q_{1}}}\right.$ for $S R R<2, t_{T d_{1}}$ for $S R R=2$ and $t_{T a_{1}}$ for $S R R>2$ ). Reference configuration with different SRRs.

Table 2: Main characteristic times involved in the transient simulations as a function of the slide-to-roll ratio (SRR). All values are given in microseconds $(\mu s)$.

\begin{tabular}{lrrrrr}
\hline SRR & 0.4 & 1.7 & 2 & 2.4 & 3.6 \\
$t_{h} / t_{T_{a}}$ & & & 60.89 & & \\
$t_{e}$ & & & 0.0288 & & \\
$t_{\dot{\gamma}}$ & 37.16 & 3.87 & 2.97 & 2.54 & 1.97 \\
$t_{T d}$ & 8.84 & 4.80 & 2.95 & 3.49 & 4.86 \\
$t_{T d_{e q_{1}}}$ & 76.11 & 405.9 & $\mathrm{x}$ & $\mathrm{x}$ & $\mathrm{x}$ \\
$t_{T d_{1}}$ & $\mathrm{x}$ & $\mathrm{x}$ & 1742 & $\mathrm{x}$ & $\mathrm{x}$ \\
$t_{T a_{1}}$ & $\mathrm{x}$ & $\mathrm{x}$ & $\mathrm{x}$ & 304.4 & 76.11 \\
\hline
\end{tabular}

Exploiting the difference in characteristic times between the different physical phenomena allows to go further into the understanding, and investigate, e.g., their relative contribution to the overall transient effects. In this event, the pure sliding case $(S R R=2)$ provides particularly helpfull information. Indeed, as the dominant thermal characteristic time $\left(t_{T d_{1}}=1742 \mu \mathrm{s}\right)$ is two order of magnitude greater than the hydrodynamic time $\left(t_{h}=60.89 \mu \mathrm{s}\right)$, their contribution to the transient effects will appear at very distinct load fluctuation frequencies. At $\Omega_{T / w}=t_{T d_{1}} / t_{w} \sim 0.029$, thermal transient effects become significant, lowering $\Delta h_{c_{m}}, \Delta A_{h_{c}}$ and $\Delta C_{f_{m}}$. Above $\Omega_{T / w} \sim 0.7$ (or $\Omega_{h / w}=t_{h} / t_{w} \sim 0.029$ ), hydrodynamic transient effects add up, progressively increasing the amplitude of the film thickness oscillations (Figure 14) as observed by Félix-Quiñonez and Morales-Espejel [40] in isothermal computations. Then, above $\Omega_{T / w} \sim 5.7\left(\Omega_{h / w} \sim 0.2\right)$, the latter completely overwhelms the former, inducing sharp modifications of the mean value of the central film thickness $\Delta h_{c_{m}}$ and of the friction coefficient $\Delta C_{f_{m}}$ (Figures 13 and 15). It is noteworthy that the delayed appearance of the hydrodynamic contribution can also be observed, though to a lower extent, for $S R R=1.7$ and $S R R=2.4$ where the dominant thermal characteristic time, respectively $t_{T d_{e q 1}}=405.9 \mu \mathrm{s}$ and $t_{T a_{1}}=304.4 \mu \mathrm{s}$, are at least 5

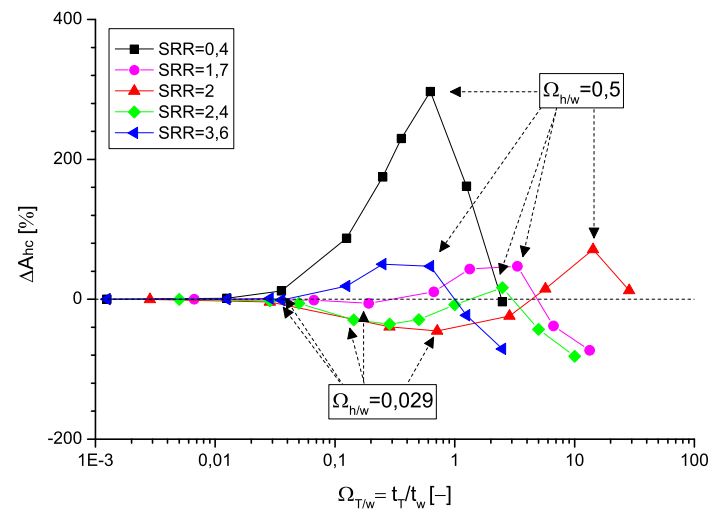

Figure 14: Influence of the transient effects on the variation of the normalized amplitude of the central film thickness oscillations $\Delta A h c$ as a function of the load fluctuation frequency $t_{w}^{-1}$. In abscissa, the latter is normalized by the dominant thermal time $t_{T}$ identified for each sliding regime $\left(t_{T d_{e q}}\right.$ for $S R R<2$, $t_{T d_{1}}$ for $S R R=2$ and $t_{T a_{1}}$ for $S R R>2$ ). Reference configuration with different SRRs.

times greater than $t_{h}=60.89 \mu \mathrm{s}$.

Finally, from a quantitative point of view, transient effects only slightly influence the average contact performance (deviations on $h_{c_{m}}$ and $C_{f_{m}}$ remain below $1.5 \%$ ). However, they tend to strongly increase the amplitude of oscillations when the ratio of the hydrodynamic time to the applied load fluctuation time $\Omega_{h / w}$ approaches 0.5 (Figure 14). Such a result has interesting practical implications in the sense that, if associated with those critical frequencies (here $t_{w}^{-1} \sim 8.2 \mathrm{kHz}$ ), large amplitude perturbations of the operating conditions may have harmful consequences, especially at low sliding.

\subsection{Influence of operating conditions and materials}

So far, the dependence of the dominant physical time (i.e. that associated with the onset of transient effects) on the slideto-roll ratio has been established using a reference configuration. In order to investigate the range of validity of the obtained expressions, a parametric study on the contact conditions was necessary. Additional computations were thus performed to test separately the influence of the amplitude of the load fluctuation $A_{w}$, the entrainment velocity $u_{0}$ and initial load $w_{0}$ and the thermal properties of the contacting bodies $\left(\rho_{i}, c_{i}, k_{i}\right)$. Those, referred to as Case 1 to 3 in the following discussion, are detailed in Table 3.

Typical results obtained at low sliding $S R R=0.4$ are reported on Figure 16. A rather good match is, again, obtained on the onset of transient effects $\left(\Omega_{T d_{e q_{1}} / w}=t_{T d_{e q_{1}}} / t_{w} \sim 0.029\right)$, implying that changes in the operating conditions or in the solid materials are either well taken into account, or without consequences. For instance, a variation of the entrainment velocity or the initial load (Case 2 on Figure 16) corresponds to the first category. Indeed, both parameters being involved in the definition of the dominant characteristic time, $t_{T d_{e q 1}}=b / u_{1}$, their modification leads to different $t_{T d_{e q_{1}}}$ but does not affect the value of $\Omega_{T d_{e q_{1}} / w}$ above which transient effects become significant. 


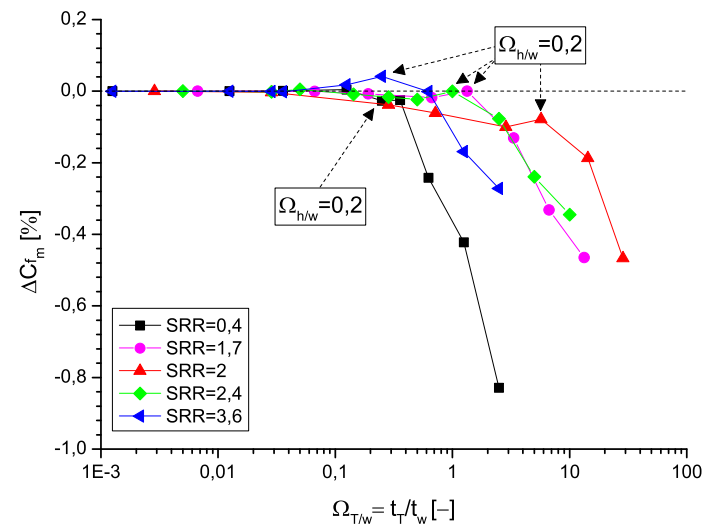

Figure 15: Influence of the transient effects on the variation of the normalized average friction coefficient $\Delta C f_{m}$ as a function of the load fluctuation frequency $t_{w}^{-1}$. In abscissa, the latter is normalized by the dominant thermal time $t_{T}$ identified for each sliding regime $\left(t_{T d_{e q_{1}}}\right.$ for $S R R<2, t_{T d_{1}}$ for $S R R=2$ and $t_{T a_{1}}$ for $S R R>2$ ). Reference configuration with different SRRs.

Table 3: Operating conditions and material properties used in the parametric study. For each computation, only the variation with the reference configuration is highlighted.

\begin{tabular}{llrrrr} 
& & Reference & Case 1 & Case 2 & Case 3 \\
\hline$A_{w}$ & $(-)$ & 0.1 & 0.2 & & \\
$u_{0}$ & $\left(\mathrm{~m} . \mathrm{s}^{-1}\right)$ & 2.5 & & 1 & \\
$w_{0}$ & $\left(\mathrm{~N} \cdot \mathrm{m}^{-1}\right)$ & 100000 & & 175000 & \\
$\rho_{i}$ & $\left(\mathrm{~kg} \cdot \mathrm{m}^{-3}\right)$ & 7850 & & & 2350 \\
$c_{i}$ & $\left(\mathrm{~J} \cdot \mathrm{kg}^{-1} . \mathrm{K}^{-1}\right)$ & 450 & & & 720 \\
$k_{i}$ & $\left(\mathrm{~W} \cdot \mathrm{m}^{-1} . \mathrm{K}^{-1}\right)$ & 47 & & & 1 \\
\hline
\end{tabular}

On the contrary, a change in the amplitude of the load fluctuation (Case 1) or in the thermal properties of the solids (Case 3) fall into the second category. Both have an obvious influence on the amplitude of the transient effects but none of them modifies $t_{T d_{e q_{1}}}$ and $\Omega_{T d_{e q_{1}} / w}$. Interestingly, this confirms the unexpected result derived from the analysis in section 3.5 according to which the onset of transient effects is independent of the solid thermal properties at low sliding (as long as the Peclet number in the slower solid, $P e_{1}=t_{T d_{e q}} / t_{T d_{1}}$ remains well above unity).

\section{Conclusion}

The paper dealt with transient effects in TEHD line contacts subjected to fluctuations of the operating conditions. An original numerical model for the simulation of such problems was first presented. Then, the phenomena at the origin of transient effects and their associated characteristic time were described. Within this framework, a particular focus was placed on the analysis of the thermal contribution. The non-trivial role of the slide-to-roll ratio (SRR) on the energy balance was highlighted, leading to the distinction of three different regimes (low, pure and high sliding). For each regime, the dominant mode of heat transfer, i.e. the most likely to give rise to transient effects, and the associated characteristic time were identified:

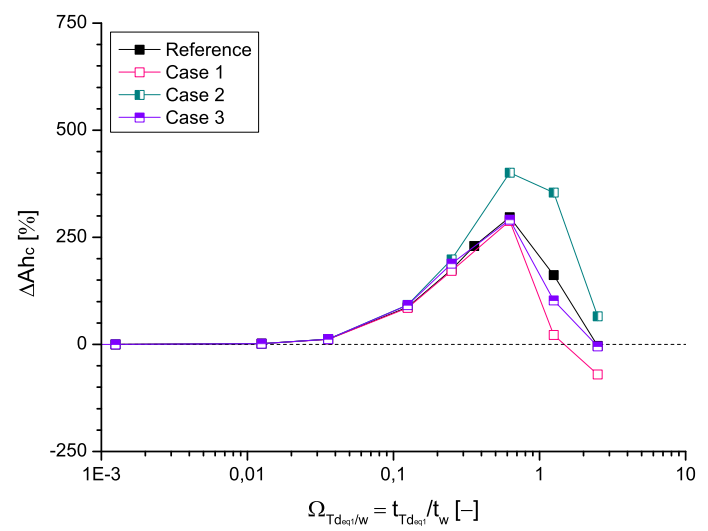

Figure 16: Influence of the transient effects on the variation of the normalized amplitude of the central film thickness oscillations $\Delta A_{h_{c}}$ as a function of the load fluctuation frequency $t_{w}^{-1}$ at low sliding $(S R R=0.4)$. In abscissa, the latter is normalized by the corresponding dominant thermal time $t_{T d_{e q_{1}}}$. Reference configuration and additional cases detailed in Table 3.

- At low sliding, the dominant thermal time corresponds to the diffusion of heat through the fluid/solid interface and into the thermal boundary layer of the slowest moving solid. Following an order of magnitude analysis, an approximate expression independent of the solid thermal properties was proposed;

- In pure sliding conditions, the dominant thermal time corresponds to the diffusion of heat through the stationary solid, from the contact center to the inlet;

- At high sliding, the dominant thermal time corresponds to the advection of heat from the contact center back to the inlet.

Then, time-dependent simulations were performed, using a reference configuration, and compared to quasisteady solutions to investigate the onset of transient effects. A direct relationship with the dominant thermal time was established and validated through a parametric study on the contact operating conditions and on the solid material properties.

The current developed approach provides an effective and convenient tool to better understand the operation of complex transient mechanical contacts. Among these, gears and camfollower systems seem to be the priority. Nonetheless, any contact featuring an unidirectional flow of lubricant (as e.g. point contacts without spinning or skewing) could also be straightforwardly studied. In addition, with the progressive increase in the use of (highly) non-Newtonian lubricants, efforts should be spent to examine the influence of the charateristic rheological time (describing the transient shear thinning behavior), which was deliberately neglected in the paper.

\section{References}

[1] W. Habchi, D. Eyheramendy, P. Vergne, G. E. Morales-Espejel, Stabilized fully-coupled finite elements for elastohydrodynamic lubrication problems, Advances in Engineering Software 46 (1) (2012) 4-18. 
[2] D. Dowson, P. Ehret, Past, present and future studies in elastohydrodynamics, Proceedings of the Institution of Mechanical Engineers, Part J: Journal of Engineering Tribology 213 (5) (1999) 317-333.

[3] D. Dowson, G. R. Higginson, Elasto-hydrodynamic lubrication: the fundamentals of roller and gear lubrication, 1st Edition, Pergamon Press (Oxford and New York), 1966.

[4] J. P. Vichard, Transient effects in the lubrication of hertzian contacts, Journal of Mechanical Engineering Science 13 (3) (1971) 173-189.

[5] M. A. Bedewi, D. Dowson, C. M. Taylor, Elastohydrodynamic lubrication of line contacts subjected to time dependent loading with particular reference to roller bearings and cams and followers, in: Proceedings of the 12th Leeds-Lyon Symposium on Tribology - Mechanisms and Surface Distress, Butterworths, London, 1985, pp. 289-304.

[6] D. Dowson, P. Harrisson, C. M. Taylor, The lubrication of automotive cams and followers, in: Proceedings of the 12th Leeds-Lyon Symposium on Tribology - Mechanisms and Surface Distress, Butterworths, London, 1985, pp. 305-322.

[7] X. Ai, H. Yu, A Numerical Analysis for the Transient EHL Process of a Cam-Tappet Pair in I. C. Engine, Journal of Tribology 111 (3) (1989) 413-417.

[8] D. Dowson, C. M. Taylor, G. Zhu, A transient elastohydrodynamic lubrication analysis of a cam and follower, Journal of Physics D: Applied Physics 25 (1A) (1992) A313-.

[9] A. W. Crook, The Lubrication of Rollers II. Film Thickness with Relation to Viscosity and Speed, Philosophical Transactions of the Royal Society of London. Series A, Mathematical and Physical Sciences 254 (1961) 223-236.

[10] A. Dyson, H. Naylor, A. R. Wilson, The measurement of oil-film thickness in elastohydrodynamic contacts, in: Proceedings of the Institution of Mechanical Engineers, Vol. 180, 1965-1966, pp. 119-134.

[11] V. Bruyere, N. Fillot, G. Morales-Espejel, P. Vergne, Computational fluid dynamics and full elasticity model for sliding line thermal elastohydrodynamic contacts, Tribology International 46 (1) (2012) 3-13.

[12] K. L. Johnson, J. L. Tevaarwerk, Shear Behaviour of Elastohydrodynamic Oil Films, Proceedings of the Royal Society of London. A. Mathematical and Physical Sciences 356 (1685) (1977) 215-236.

[13] S. Bair, W. O. Winer, A Rheological Model for Elastohydrodynamic Contacts Based on Primary Laboratory Data, Journal of Lubrication Technology 101 (3) (1979) 258-264.

[14] K. L. Johnson, J. A. Greenwood, Thermal analysis of an Eyring fluid in elastohydrodynamic traction, Wear 61 (2) (1980) 353-374.

[15] L. Houpert, L. Flamand, D. Berthe, Rheological and Thermal Effects in Lubricated E.H.D. Contacts, Journal of Lubrication Technology 103 (4) (1981) 526-532.

[16] A. Brandt, A. A. Lubrecht, Multilevel matrix multiplication and fast solution of integral equations, Journal of Computational Physics 90 (2) (1990) $348-370$.

[17] C. H. Venner, A. A. Lubrecht, W. E. ten Napel, Numerical Simulation of the Overrolling of a Surface Feature in an EHL Line Contact, Journal of Tribology 113 (4) (1991) 777-783.

[18] R. Larsson, Transient non-Newtonian elastohydrodynamic lubrication analysis of an involute spur gear, Wear 207 (12) (1997) 67-73.

[19] J. Wang, P. Yang, A Numerical Analysis for TEHL of Eccentric-Tappet Pair Subjected to Transient Load, Journal of Tribology 125 (4) (2003) 770-779.

[20] A. Cameron, The Viscosity Wedge, A S L E Transactions 1 (2) (1958) 248-253.

[21] L. Bobach, R. Beilicke, D. Bartel, L. Deters, Thermal elastohydrodynamic simulation of involute spur gears incorporating mixed friction, Tribology International 48 (0) (2012) 191-206.

[22] T. Doki-Thonon, Thermal effects in elastohydrodynamic spinning circular contacts, Ph.D. thesis, INSA de Lyon (2012).

URL http: //tel.archives-ouvertes.fr/tel-00749882

[23] W. Habchi, A full-system finite element approach to elastohydrodynamic lubrication problems: application to ultra-low-viscosity fluids, Ph.D. thesis, INSA de Lyon (2008).

URL http://theses.insa-lyon.fr/publication/ 2008ISAL0038/these.pdf

[24] P. Yang, S. Wen, A Generalized Reynolds Equation for Non-Newtonian Thermal Elastohydrodynamic Lubrication, Journal of Tribology 112 (4) (1990) 631-636.
[25] P. Yang, Z. M. Jin, F. Liu, D. Dowson, On the time-dependent, thermal and non-Newtonian elastohydrodynamic lubrication of line contacts subjected to normal and tangential vibrations, Proceedings of the Institution of Mechanical Engineers, Part J: Journal of Engineering Tribology 218 (2) (2004) 71-82.

[26] S. Yasutomi, S. Bair, W. O. Winer, An Application of a Free Volume Model to Lubricant Rheology I-Dependence of Viscosity on Temperature and Pressure, Journal of Tribology 106 (2) (1984) 291-302.

[27] S. Bair, A Rough Shear-Thinning Correction for EHD Film Thickness, Tribology Transactions 47 (3) (2004) 361-365.

[28] F. P. Incropera, D. P. DeWitt, Fundamentals of Heat and Mass Transfer, John Wiley \& Sons, 1996.

[29] Comsol, COMSOL Multiphysics Reference Guide version 4.1 (2010).

[30] C. Venner, F. Couhier, A. Lubrecht, J. Greenwood, Amplitude Reduction of Waviness in Transient EHL Line Contacts, in: Elastohydrodynamics - '96 Fundamentals and Applications in Lubrication and Traction, Proceedings of the 23rd Leeds-Lyon Symposium on Tribology, Vol. Volume 32, Elsevier, 1997, pp. 103-112.

[31] C. Hooke, Surface roughness modification in EHL line contacts-the effect of roughness wavelength, orientation and operating conditions, in: Lubrication at the Frontier The Role of the Interface and Surface Layers in the Thin Film and Boundary Regime, Proceedings of the 25th Leeds-Lyon Symposium on Tribology, Vol. Volume 36, Elsevier, 1999, pp. 193-202.

[32] C. J. Hooke, C. H. Venner, Surface roughness attenuation in line and point contacts, Proceedings of the Institution of Mechanical Engineers, Part J: Journal of Engineering Tribology 214 (5) (2000) 439-444.

[33] J. Achenbach, Wave propagation in elastic solids, North-Holland Pub. Co., 1973.

[34] P. C. Vinh, R. Ogden, On formulas for the Rayleigh wave speed, Wave Motion 39 (3) (2004) 191-197.

[35] R. Bird, O. Hassager, Dynamics of Polymeric Liquids: Fluid mechanics, Wiley, 1987.

[36] W. Habchi, S. Bair, P. Vergne, On friction regimes in quantitative elastohydrodynamics, Tribology International 58 (0) (2013) 107-117.

[37] C. Rauwendaal, Polymer extrusion, Hanser Verlag, 2001

[38] M. Thirumaleshwar, Fundamentals of Heat and Mass Transfer, Pearson Education, 2006.

[39] L. Jiji, Heat Convection, Springer-Verlag Berlin Heidelberg, 2009.

[40] A. Félix-Quiñonez, G. E. Morales-Espejel, Film thickness fluctuations in time-varying normal loading of rolling elastohydrodynamically lubricated contacts, Proceedings of the Institution of Mechanical Engineers, Part C: Journal of Mechanical Engineering Science 224 (12) (2010) 2559-2567. 


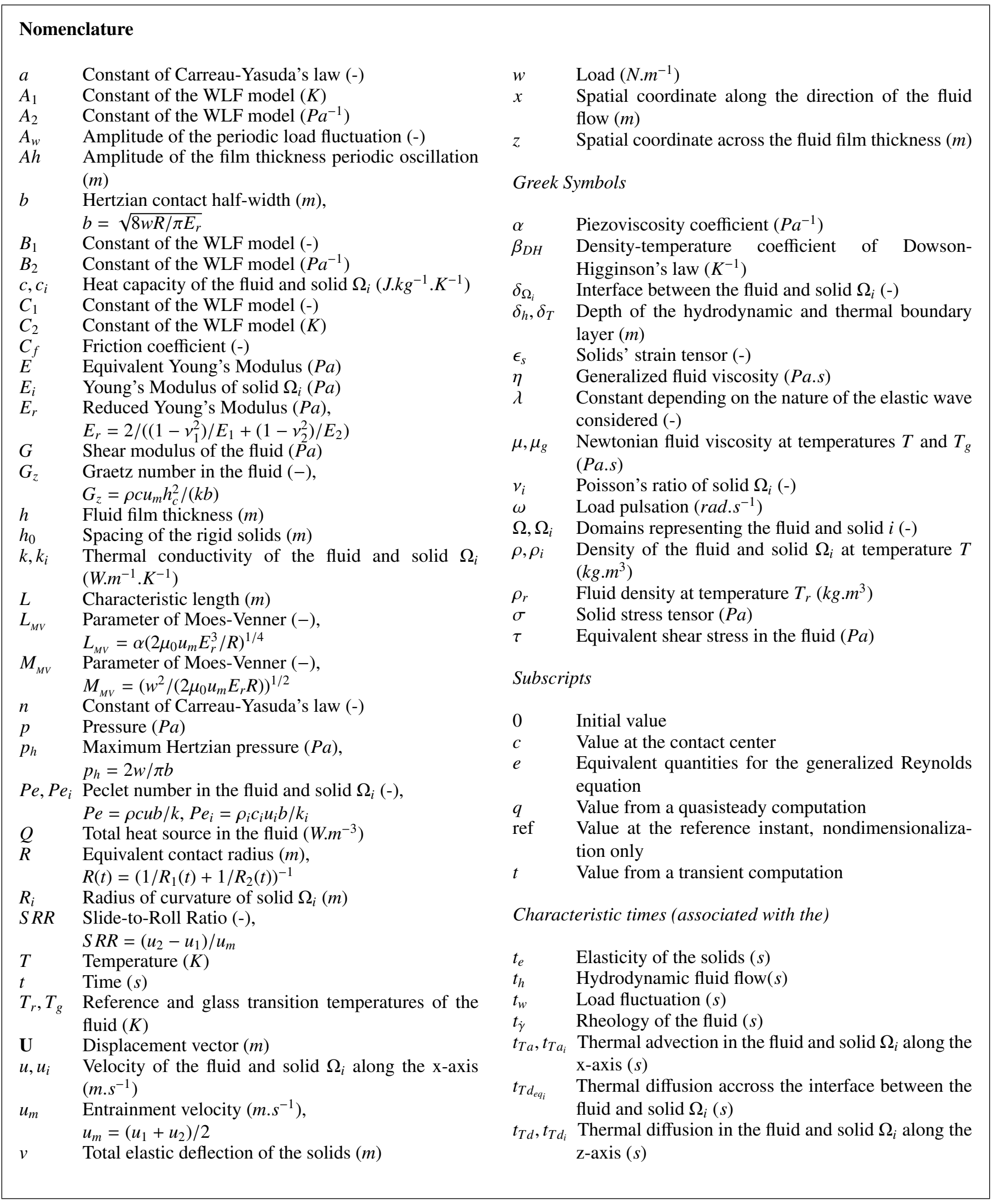

